\title{
Investigation of clinical diagnosis and liver biopsy diagnosis in cases of patients with chronic HBV infection
}

\author{
W.-Q. Shi, W. Ni and Y.-L. Yang \\ Liver Diseases Bureau, \\ Second Affiliated Hospital of Zhejiang Chinese Medical University, \\ Hangzhou, China \\ Corresponding author: W. Ni \\ E-mail: niweicn@yeah.net
}

Genet. Mol. Res. 13 (2): 2931-2938 (2014)

Received July 14, 2013

Accepted December 13, 2013

Published February 13, 2014

DOI http://dx.doi.org/10.4238/2014.February.13.16

\begin{abstract}
The aim of this study was to investigate the pathomorphological changes of chronic hepatitis B virus (HBV) infection and the coincidence between clinical and pathological diagnosis. Hematoxylin and eosin staining and immunohistochemistry for HBsAg and $\mathrm{HBcAg}$ were performed on 97 liver biopsy specimens collected from 97 patients with chronic HBV infection, including $55 \mathrm{HBV}$ carriers. The agreement between clinical diagnosis and pathological diagnosis in mild, moderate and severe chronic hepatitis was $71.4,60$ and $83.3 \%$. The rate of clinical diagnosis matching the pathological diagnosis for fibrosis was higher than for inflammation ( 83.33 vs $54.76 \%$ ), especially in moderate ( 83.33 vs $26.67 \%$ ) and severe chronic hepatitis (66.67 vs 16.67\%). The rate of serological/imaging examination results and immunohistochemistry confirmation was as high as $93.81 \%$ for $\mathrm{HBsAg}$ and $92.78 \%$ for $\mathrm{HBcAg}$. In HBV carriers, the degree of inflammation and fibrosis in most cases was mild, and the younger the patients, the higher the ratios of G0 and S0. The clinical diagnosis was correct in most cases, especially for inflammation and in mild chronic hepatitis; the patients who were diagnosed as HBV
\end{abstract}


carriers actually already had inflammation and fibrosis.

Key words: Liver biopsy; Clinical diagnosis; Pathological diagnosis; Immunohistochemistry; HBV infection

\section{INTRODUCTION}

Hepatitis B virus (HBV) infection is a serious global public health problem affecting billions of people globally (Alavian et al., 2012), where it is said that $1 / 3$ of all people in the world have been infected with HBV at some time. The prevalence of chronic hepatitis B (CHB) has tended to increase in recent years (Hadziyannis and Papatheodoridis, 2006), where it is reported that there are 350 million people diagnosed with CHB (Fox, 2012) and that 1 million die of disease caused by HBV every year (Abdo et al., 2012). In China, the ratio of HBsAg-positive infection was 9.75\% in 1992 ( $\mathrm{Su}$ and Ye, 2012), which was defined as high prevalence, but it decreased in recent decades with popularity of HBV vaccination and chemotherapy (Hwang et al., 2012). Although the prevalence and incidence have declined now, the total number of HBV infection is still very large (Yang et al., 2012).

Most of the time, HBV diagnosis depends on clinical symptoms and blood tests, including $\mathrm{HBV}$ antigen (HBsAg/HBeAg) or antibody (HBsAb/HBeAb/HBcAb) detection and liver enzyme (usually including alanine aminotransferase (ALT) and aspartate aminotransferase (AST) assays. When the result of the antigen test is negative, serum liver enzymes are frequently determined to aid disease diagnosis (Hann et al., 2012), and usually there is a positive correlation between serum ALT or AST level and hepatic inflammation and fibrosis in patients with HBV infection (Zheng et al., 2010; Bai et al., 2013). However, there are some patients whose HBV antigen test is negative and the levels of AST and ALT are not very high. An earlier prospective study showed that in the patients infected with HBV whose ALT was $\leq$ twice upper limit of normal (ULN), 59.3\% had significant hepatic inflammation and $62.1 \%$ had significant hepatic fibrosis (Lesmana et al., 2011). Therefore, pathological diagnosis is still the most effective and gold standard diagnostic method for HBV, especially for serodiagnosisnegative patients (Puoti, 2013). With liver biopsy, the diagnostic accuracy would increase and the degree of inflammation and fibrosis could be accurately predicated.

In this study, 97 cases of liver biopsies from chronic HBV infection patients in the Liver Diseases Bureau, Second Affiliated Hospital of Zhejiang Chinese Medical University, were recruit and were grouped into 17-29, 30-40 and 41-57 years. The slides were collected to investigate the coincidence of clinical and pathological diagnosis, and the degree of inflammation and fibrosis in the different age groups.

\section{MATERIAL AND METHODS}

\section{Subjects}

Ninety-seven cases were inpatients including 57 males and 40 females. This study was conducted in accordance with the Declaration of Helsinki and with the approval of the Ethics Committee of the Second Affiliated Hospital of Zhejiang Chinese Medical University, and written informed consent was obtained from all participants. Their ages varied from 17 to 57 years and the mean age was 33.6 years. The course of disease varied from 11 months to 
33 years. According to the diagnostic criteria revised during the National Conference of Viral Hepatitis in 2000 (Chinese Society of Infectious and Parasitic Diseases, Hepatology, 2000), 97 cases of patients with clinical diagnosis were summarized as follows: 55 cases of HBV carriers, 42 cases of chronic hepatitis B patients (according to clinical mild, moderate and severe classification, there were 21,15 and 16 cases, respectively), among which there were 3 cases of $\mathrm{HBV} / \mathrm{HEV}$ co-infection and 2 cases of $\mathrm{HBV} / \mathrm{HCV}$ co-infection. Pathological diagnosis was based on the standard revised in the National Conference of Parasitic and Infectious Diseases in 2000 (Chinese Society of Infectious and Parasitic Diseases, Hepatology, 2000): mild chronic hepatitis G1-2, S0-2; moderate chronic hepatitis G3, S2-3; severe chronic hepatitis G4, S3-4. All patients had not received antiviral therapy before biopsy.

A liver tissue specimen (length $>1.0 \mathrm{~cm}$ ) was obtained by introducing the needle into the liver substance and withdrawing rapidly within $1 \mathrm{~s}$. The biopsy was fixed in $10 \%$ formal saline, conventionally dehydrated, sliced, embedded in paraffin, and stained using hematoxylin and eosin. Immunohistochemical double staining with detection of Flag-tagged proteins ( $\mathrm{HBsAg}$, $\mathrm{HBcAg}$ ) was observed with a light microscope. With the consent of their families, two patients had liver biopsy when approaching clinical death or $2 \mathrm{~h}$ after the clinical diagnosis of death.

\section{RESULTS}

\section{Comparison of the clinical and pathological diagnosis}

In the majority of cases, clinical and pathological diagnosis were in agreement. For example, $87.3 \%$ of the HBV carriers were confirmed to have mild chronic hepatitis by pathological diagnosis. Clinical diagnosis agreed with pathological diagnosis for mild, moderate and severe chronic hepatitis in 71.4, 60 and $83.3 \%$ of cases, so we concluded that the accuracy of clinical diagnosis for moderate chronic hepatitis was lower than for mild or severe chronic hepatitis (Table 1).

\begin{tabular}{|c|c|c|c|c|}
\hline \multirow[t]{2}{*}{ Clinical diagnosis } & \multirow[t]{2}{*}{ Cases } & \multicolumn{3}{|c|}{ Pathological diagnosis (cases) } \\
\hline & & Mild CH & Moderate $\mathrm{CH}$ & Severe $\mathrm{CH}$ \\
\hline HBV carriers & 55 & 48 & 5 & 2 \\
\hline Mild CH & 21 & 15 & 4 & 2 \\
\hline Moderate $\mathrm{CH}$ & 15 & 2 & 9 & 4 \\
\hline Severe $\mathrm{CH}$ & 6 & 0 & 1 & 5 \\
\hline
\end{tabular}

$\mathrm{CH}=$ chronic hepatitis.

\section{Comparison of the degree of inflammation and fibrosis by clinical and pathological diagnosis}

The total coincidence was $83.33 \%$. The results of mild chronic hepatitis showed that 95.24\% (20/21) of the clinical diagnosis matched the pathological diagnosis, and in severe chronic hepatitis the agreement was $83.33 \%(5 / 6)$, while the rate of diagnostic confirmation in moderate chronic hepatitis was the lowest, only $66.67 \%(10 / 15)$ (Table 2). 
Table 2. Comparison of the degree of fibrosis by clinical and pathological diagnosis among 97 cases of patients with HBV infection.

\begin{tabular}{|c|c|c|c|c|c|c|}
\hline \multirow[t]{2}{*}{ Clinical diagnosis } & \multirow[t]{2}{*}{ Cases } & \multicolumn{5}{|c|}{ Pathological diagnosis (cases) } \\
\hline & & So & S1 & S2 & S3 & S4 \\
\hline HBV carriers & 55 & 31 & 21 & 2 & 1 & 0 \\
\hline Mild CH & 21 & 6 & 12 & 2 & 1 & 0 \\
\hline Moderate $\mathrm{CH}$ & 15 & 3 & 2 & 8 & 2 & 0 \\
\hline Severe $\mathrm{CH}$ & 6 & 0 & 0 & 1 & 4 & 1 \\
\hline
\end{tabular}

$\mathrm{CH}=$ chronic hepatitis.

For inflammation, the total coincidence was $54,76 \%$. The results for mild chronic hepatitis showed that clinical diagnosis matched pathological diagnosis in $85.71 \%$ of cases $(18 / 21)$, but the confirmation rate in moderate and severe chronic hepatitis was much lower, where it was respectively only $26.67 \%(4 / 15)$ and $16.67 \%(1 / 6)$ (Table 3$)$.

\begin{tabular}{|c|c|c|c|c|c|c|}
\hline \multirow{2}{*}{ Clinical diagnosis } & \multirow[t]{2}{*}{ Cases } & \multicolumn{5}{|c|}{ Pathological diagnosis (cases) } \\
\hline & & G0 & G1 & G2 & G3 & $\mathrm{G} 4$ \\
\hline HBV carriers & 55 & 28 & 23 & 3 & 1 & 0 \\
\hline Mild CH & 21 & 1 & 11 & 7 & 2 & 0 \\
\hline Moderate $\mathrm{CH}$ & 15 & 1 & 2 & 8 & 4 & 0 \\
\hline Severe CH & 6 & 0 & 0 & 2 & 3 & 1 \\
\hline
\end{tabular}

$\mathrm{CH}=$ chronic hepatitis.

\section{Comparison of clinical diagnosis and immunohistochemistry of liver biopsy}

Among the 97 patients who were diagnosed with chronic HBV infection, 93.81\% (91/97) biopsy specimens expressed HBsAg and 92.78\% (90/97) expressed HBcAg (Table 4).

Table 4. Expression of HBsAg and HBcAg in liver tissues among 97 cases of patients with chronic HBV infection by clinical diagnosis.

\begin{tabular}{lccc}
\hline Liver biopsy results & Cases & Positive & Negative \\
\hline HBsAg & 97 & 91 & 6 \\
HBcAg & 97 & 90 & 7 \\
\hline
\end{tabular}

\section{Analysis of liver histopathological diagnosis among 55 cases of chronic $\mathrm{HBV}$ carriers categorized by age}

Among the 55 cases of chronic HBV carriers, the degree of inflammation in most cases indicated mild chronic hepatitis at all ages, and there was only 1 case in the 41-57 years group that was G3, which is moderate. The younger the patients, the lower the degree of inflammation. In the 17-29-year group, 62.25\% were G0, but in the older group, the rates of G0 and G1 were close. It should be noted that in the 30-40-year group, there were 32 cases, but in the 17-29- and 41-57-year groups, there were respectively only 8 and 14 cases, so it was possible that this result did not reflect the real situation (Table 5). 
Table 5. Comparison of the degree of inflammation among 55 cases of chronic HBV carriers categorized by age through pathological diagnosis.

\begin{tabular}{|c|c|c|c|c|c|c|}
\hline & \multicolumn{2}{|c|}{$17-29$ years } & \multicolumn{2}{|c|}{$30-40$ years } & \multicolumn{2}{|c|}{$41-57$ years } \\
\hline & Cases & Ratio (\%) & Cases & Ratio (\%) & Cases & Ratio (\%) \\
\hline G0 & 5 & 62.5 & 15 & 46.9 & 8 & 53.3 \\
\hline G1 & 2 & 25.0 & 16 & 50.0 & 5 & 33.3 \\
\hline G2 & 1 & 12.5 & 1 & 3.1 & 1 & 6.7 \\
\hline G3 & 0 & 0.0 & 0 & 0.0 & 1 & 6.7 \\
\hline G4 & 0 & 0.0 & 0 & 0.0 & 0 & 0.0 \\
\hline Total & 8 & & 32 & & 15 & \\
\hline
\end{tabular}

The result showed the correspondence between S and G. Similarly, the rate of S0 was much higher in the younger group. Among the 8 cases who were HBV carriers, $6(75.0 \%)$ were $\mathrm{S} 0$, while in the middle age group, the ratio of S0 was $59.4 \%$ and $\mathrm{S} 1$ was $37.5 \%$, in the older group, the rate was 40.0 and $46.7 \%$, respectively. In both the middle age and older groups, the rates of S0 and S1 were much closer. Notably, only in the older group were there 2 cases of $\mathrm{S} 2$ or $\mathrm{S} 3$, which means moderate to severe hepatitis. But again, there may be bias in the result since the cases in the younger and older groups were not sufficient (Table 6).

Table 6. Comparison of the degree of fibrosis among 55 cases of patients pathologically diagnosed as chronic HBV carriers classified by age.

\begin{tabular}{|c|c|c|c|c|c|c|}
\hline & \multicolumn{2}{|c|}{$17-29$ years } & \multicolumn{2}{|c|}{$30-40$ years } & \multicolumn{2}{|c|}{$41-57$ years } \\
\hline & Cases & Ratio (\%) & Cases & Ratio (\%) & Cases & Ratio (\%) \\
\hline S0 & 6 & 75.0 & 19 & 59.4 & 6 & 40.0 \\
\hline $\mathrm{S} 1$ & 2 & 25.0 & 12 & 37.5 & 7 & 46.7 \\
\hline S2 & 0 & 0.0 & 1 & 3.1 & 1 & 6.7 \\
\hline S3 & 0 & 0.0 & 0 & 0.0 & 1 & 6.7 \\
\hline S4 & 0 & 0.0 & 0 & 0.0 & 0 & 0 \\
\hline Total & 8 & & 32 & & 15 & \\
\hline
\end{tabular}

\section{DISCUSSION}

The diagnosis of chronic liver disease relies on clinical symptoms, signs, serological tests, and imaging examinations, but many patients with chronic liver disease have no obvious clinical manifestations or signs (Okuse et al., 2012). Even serological examination and imaging examination cannot reflect the real situation of the liver. These bring about many difficulties in diagnosis, treatment and judgment of prognosis. Despite the fact that liver biopsy is not easily acceptable and limited to some extent, it is still recognized as the gold standard for the diagnosis of liver lesions at home and abroad (Cheong et al., 2012). Implementation of the liver biopsy is not only able to grasp the morphological changes of the liver tissue, but also may get more valuable tips identifying the degree of inflammation and fibrosis in the liver and the unknown liver damage (Ren et al., 2013), which will individualize therapeutic schemes, predicting the effects of treatment (Cheng et al., 2005). Hence, liver biopsy should be strongly advocated in clinical work.

Recently, there have been considerable interests in chronic HBV carriers at home and abroad. The number of controversies around early intervention of HBV carriers has been on the rise (Bottero et al., 2013). Although there is no consensus on this issue, most authors still 
advocate that this part of the population should be reexamined and followed-up periodically without anti-viral treatment (Norouzi et al., 2012). There were 55 cases that were diagnosed as HBV carriers through clinical diagnosis: 24 cases of patients had fibrosis (21 S1, 2 S2, 1 S3) and 31 cases of patients had no fibrosis (S0); 27 cases of patients had inflammation ( $23 \mathrm{G} 1$, $3 \mathrm{G} 2,1 \mathrm{G} 3$ ) and 28 cases had no inflammation (G0). The above results suggest that although chronic HBV carriers were in stable conditions without inflammation and fibrosis according to serological tests, serological tests cannot fully reflect the real situation of the liver.

Fifty-five cases of patients clinically diagnosed as HBV carriers were divided into three groups according to age: 17-29, 30-40 and 41-57 years. Comparing with the results of pathological diagnosis, respectively, the degree of liver inflammation and fibrosis was more severe with increasing age. In the 17-29-year group, the percentages of patients without significant inflammation and fibrosis were $62.5 \%(\mathrm{G} 0)$ and $75.0 \%(\mathrm{~S} 0)$, respectively. In the 30 40-year group, the percentages of patients with significant inflammation and fibrosis were $53.1 \%(\mathrm{G} 1: 50.0 \%, \mathrm{G} 2: 3.1 \%)$ and $40.6 \%$ (S1: $37.5 \%$, S2: 3.1\%). In the 41-57-year group, the percentages of patients with significant inflammation and fibrosis were $46.7 \%$ (G1: $33.3 \%$, G2: 6.7\%, G3: 6.7\%) and 60.1\% (S1: 46.7\%, S2: 6.7\%, S3: 6.7\%), respectively. Comparing the 30-40- and 41-57-year groups, the percentage of patients with inflammation was higher in the latter, while the percentage of patients with fibrosis was higher than the former. This may have something to do with the infection status of chronic HBV. The younger the patients, the greater the inflammatory reactions. However, the inflammatory reactions lessened and the degree of fibrosis increased with increasing age. Therefore, it is believed that persistently normal liver function should not become a barrier to antiviral treatment and that liver biopsy is equally important for chronic HBV, especially, placing emphasis on liver biopsy of chronic HBV carriers who are more than 30 years old, which would guarantee understanding the disease and timely intervention. When chronic HBV carriers had significant inflammation and fibrosis after liver biopsy, it was necessary to intervene in a timely manner.

Forty-two cases of patients clinically diagnosed with mild, moderate or severe chronic HBV and patients pathologically diagnosed with fibrosis and inflammation were not closely related. Among the liver biopsy results clinically diagnosed as the same type of chronic hepatitis $\mathrm{B}$, the number of pathological diagnosed cases was more than that of clinical diagnosis, regardless of the degree of inflammation and fibrosis. The above results once again revealed the limitations of serological tests or imaging examinations, concealing the seriousness of some patients with liver inflammation and fibrosis, which may be related to the liver having a strong compensatory ability. During the period of chronic inflammatory liver, the rise in serum ALT and AST means lesions of the unit membrane of liver cells. ALT and AST levels indicate damage to the surface of liver cells instead of the degree of damage (Zhu et al., 2011). They are ineffective in determining the degree of liver inflammation only by their rise. The degree of inflammation shown by the liver biopsy has been divided into several stages based on inflammation of portal area and acinar inflammation and necrosis (Papatheodoridis et al., 2012). Especially, in the stage of severe hepatitis because of liver cell necrosis, ALT and AST were not increased. Therefore, liver biopsy can reveal more accurately the degree of inflammation and fibrosis of the liver parenchyma (Chu et al., 2013). There were some differences in the degree of inflammation and fibrosis between the two methods. Although liver biopsy is universally accepted as the gold standard, it is in need of some conditions to realize its diagnostic accuracy.

Among 97 cases of patients clinically diagnosed with chronic HBV infection, liver 
biopsy suggested that there were 6 cases of $\mathrm{HBsAg}$-negative and 7 cases of $\mathrm{HBcAg}$-negative, which may have something to do with uneven liver inflammation and fibrosis. Meanwhile, as an invasive operation, some external factors such as the length of sample, diameter and timeliness should be taken into consideration when performing a liver biopsy. These external factors could have an impact on the results. It is necessary to have both liver biopsy and immunohistochemical examination done at the same time.

Clinicians should be concerned about people with chronic infection, including chronic HBV carriers, and fully aware of the need of liver biopsy for this group. In addition to the conventional serological tests and imaging examination, we should pay attention to the role of liver biopsy plays in the diagnosis of liver diseases and selection of therapeutic regimens. We can accurately determine the degree of liver inflammation and fibrosis through liver biopsy, which can provide the basis for individualized treatment. If necessary, liver biopsy can be performed several times.

\section{REFERENCES}

Abdo AA, Sanai FM and Al-Faleh FZ (2012). Epidemiology of viral hepatitis in Saudi Arabia: are we off the hook? Saudi J. Gastroenterol. 18: 349-357.

Alavian SM, Tabatabaei SV, Ghadimi T, Beedrapour F, et al. (2012). Seroprevalence of hepatitis B virus infection and its risk factors in the West of Iran: A Population-Based Study. Int. J. Prev. Med. 3: 770-775.

Bai H, Liu H, Chen X, Xu C, et al. (2013). Influence of age and HBeAg status on the correlation between HBV DNA and hepatic inflammation and fibrosis in chronic hepatitis B patients. Dig. Dis. Sci. 58: 1355-1362.

Bottero J, Boyd A, Gozlan J, Lemoine M, et al. (2013). Performance of rapid tests for detection of HBsAg and anti-HBsAb in a large cohort, France. J. Hepatol. 58: 473-478.

Cheng HC, Sun L, Liu P and Xu XP (2005). Clinical analysis of 4449 cases of liver biopsy. Med. J. Chin. People's Liberation Army 30: 319.

Cheong JY, Um SH, Seo YS, Shin SS, et al. (2012). A practical scoring system for predicting cirrhosis in patients with chronic viral hepatitis. Hepatogastroenterology 59: 2592-2597.

Chinese Society of Infectious and Parasitic Diseases Hepatology (2000). Viral hepatitis control schemes. Chin. J. Hepatol. 8: 324-329.

Chu CM, Lin DY and Liaw YF (2013). Clinical and virological characteristics post HBsAg seroclearance in hepatitis B virus carriers with hepatic steatosis versus those without. Dig. Dis. Sci. 58: 275-281.

Fox R (2012). Hepatitis B Infection. Guide for HIV/AIDS Clinical Care, HRSA HIV/AIDS Bureau.

Hadziyannis SJ and Papatheodoridis GV (2006). Hepatitis B e antigen-negative chronic hepatitis B: natural history and treatment. Semin. Liver Dis. 26: 130-141.

Hann HW, Wan S, Myers RE, Hann RS, et al. (2012). Comprehensive analysis of common serum liver enzymes as prospective predictors of hepatocellular carcinoma in HBV patients. PLoS One 7: e47687.

Hwang JP, Fisch MJ, Zhang H, Kallen MA, et al. (2012). Low rates of hepatitis B virus screening at the onset of chemotherapy. J. Oncol. Pract. 8: e32-e39.

Lesmana CR, Gani RA, Hasan I, Simadibrata M, et al. (2011). Significant hepatic histopathology in chronic hepatitis B patients with serum ALT less than twice ULN and high HBV-DNA levels in Indonesia. J. Dig. Dis. 12: 476-480.

Norouzi M, Ghorashi S, Abedi F and Nejatizadeh A (2012). Identification of hepatitis B virus surface antigen (HBsAg) genotypes and variations in chronic carriers from Isfahan Province, Iran. Iran. J. Public. Health 41: 104-111.

Okuse C, Yotsuyanagi H, Yamada N, Ikeda H, et al. (2012). Changes in levels of hepatitis B virus markers in patients positive for low-titer hepatitis B surface antigen. Hepatol. Res. 42: 1236-1240.

Papatheodoridis GV, Manolakopoulos S, Liaw YF and Lok A (2012). Follow-up and indications for liver biopsy in HBeAg-negative chronic hepatitis B virus infection with persistently normal ALT: a systematic review. J. Hepatol. 57: 196-202.

Puoti C (2013). How to manage HBeAg-negative chronic HBV infection with normal alanine aminotransferase levels in clinical practice? Eur. J. Intern. Med. 24: 100-103.

Ren YY, Liu YZ, Ding YP, Song G, et al. (2013). Immune characteristics of different immune phases in natural course of chronic HBV infection. Hepatogastroenterology 60: 789-795. 
Su QM and Ye XG (2012). Effects of telbivudine and entecavir for HBeAg-positive chronic hepatitis B: a meta-analysis. World J. Gastroenterol. 18: 6290-6301.

Yang SG, Wang B, Chen P, Yu CB, et al. (2012). Effectiveness of HBV vaccination in infants and prediction of HBV prevalence trend under new vaccination plan: findings of a large-scale investigation. PLoS One 7: e47808.

Zheng RD, Xu CR, Jiang L, Dou AX, et al. (2010). Predictors of hepatic steatosis in HBeAg-negative chronic hepatitis B patients and their diagnostic values in hepatic fibrosis. Int. J. Med. Sci. 7: 272-277.

Zhu X, Wang LC, Chen EQ, Chen XB, et al. (2011). Prospective evaluation of FibroScan for the diagnosis of hepatic fibrosis compared with liver biopsy/AST platelet ratio index and FIB-4 in patients with chronic HBV infection. Dig. Dis. Sci. 56: 2742-2749. 